\title{
Seletividade de sulfentrazone aplicado em pré-plantio incorporado e em pré- emergência da cultura da cana-de-açúcar
}

\author{
Gustavo Soares da Silva ${ }^{1}$, André Felipe Moreira Silva ${ }^{1}$, Luiz Henrique Freguglia Aiello ${ }^{2}$, Roberto Estêvão \\ Bragion de Toledo ${ }^{1}$, Giovani Apolari Ghirardello ${ }^{1}$, Ricardo Victoria Filho ${ }^{1}$ \\ ${ }^{1}$ Universidade de São Paulo, Escola Superior de Agricultura "Luiz de Queiroz" - Esalq, Piracicaba, SP. ${ }^{2}$ Associação Mata \\ Ciliar, Jundiaí - SP. E-mail: gustavusoares@gmail.com
}

\section{Resumo}

Os herbicidas podem ser seletivos ou não a cana-de-açúcar, aplicados em pré-plantio incorporado (PPI) e pré-emergência (PRÉ) das plantas daninhas e da cultura. O objetivo do trabalho foi avaliar a seletividade do sulfentrazone, aplicado tanto em pré-plantio incorporado, como em pré-emergência das seguintes variedades de cana-de-açúcar: CTC 9001, CTC 9002, CTC 9003 e CTC 14. O experimento foi conduzido em casa-de-vegetação no delineamento inteiramente casualizado com quatro repetições. Os tratamentos eram constituídos por quatro cultivares de cana-de-açúcar, submetidas a seis doses de sulfentrazone $(0,100$, 200, 400, 800, 1600 g i.a. ha ${ }^{-1}$ ), tanto em PRÉ como em PPI. As avaliações de fitotoxicidade foram realizadas aos 30, 37, 44, 51 e 58 DAA e nesta última foi feita avaliação de massa seca da parte aérea. 0 sulfentrazone aplicado em PRÉ foi seletivo até a dose de $400 \mathrm{~g}$ i.a. ha ${ }^{-1}$, apresentando sintomas de fitotoxicidade nas doses superiores. Em PPI, os sintomas de fitotoxicidade foram verificados a partir da dose de $400 \mathrm{~g}$ i.a. ha ${ }^{-1}$ A variedade CTC 9002 obteve maior acúmulo de massa seca tanto em PRÉ quanto em PPI. A CTC 14 tolera maiores doses de sulfentrazone em PRÉ e a CTC 9001, em PPI, tendo a massa seca menos prejudicada.

Palavras-chave: Saccharum officcinarum; herbicida no solo; tolerância a herbicidas.

Selectivity of sulfentrazone applied in pre-planting incorporated and in pre-emergence of sugarcane crop

\begin{abstract}
Herbicides can be selective or non-selective to sugarcane, applied both in pre-planting incorporated (PPI) and pre-emergence (PRE) of weeds and crop. The aim of the study was to evaluate the selectivity of sulfentrazone, applied in pre-planting and pre-emergence of the following sugarcane varieties: CTC 9001, CTC 9002, CTC 9003 and CTC 14. The experiment was carried out in a greenhouse with a completely randomized design with four replications. The treatments were composed of four sugarcane varieties, submitted to six doses of sulfentrazone $\left(0,100,200,400,800,1600 \mathrm{~g}\right.$ a.i. ha $\left.{ }^{-1}\right)$, applied both in PRE and PPI. The phytotoxicity evaluations were performed at 30,37, 44, 51 and 58 DAA and the aerial dry mass was evaluated at 58 DAA. The sulfentrazone applied in PRE was selective up to the $400 \mathrm{~g}$ a.i. $\mathrm{ha}^{-1}$ dose, showing more symptoms at higher rates. In PPI, the symptoms of phytotoxicity were verified from the dose of $400 \mathrm{~g}$ a.i. ha ${ }^{-1}$. The CTC 9002 variety presented a higher dry mass accumulation both in PRE and PPI. CTC 14 variety tolerated higher doses of sulfentrazone in PRE and the CTC 9001 in PPI, both having a lower decrease on its dry mass values.
\end{abstract}

Keywords: Saccharum officcinarum; soil herbicide; herbicide tolerance.

\section{Introdução}

Um dos pontos mais críticos no processo produtivo da cana-de-açúcar, tanto em áreas de plantio como em áreas de cana soca, é a capacidade de interferência no desenvolvimento e na produtividade imposta pelas plantas daninhas (TOLEDO et al., 2015). As plantas daninhas podem prejudicar o desenvolvimento inicial e a produtividade do cultivo, por competirem pelos recursos disponíveis do meio, 
além de causarem problemas na fase de corte e colheita, e encurtarem a vida útil do canavial (VICTORIA FILHO; CHRISTOFFOLETI, 2004; KUVA et al., 2007).

O uso de herbicidas é uma importante ferramenta no manejo de plantas daninhas. Estes podem se apresentar como seletivos ou não ao cultivo, e podem ser classificados nas seguintes modalidades de aplicação: Pré-plantio incorporado (PPI), pré-emergência (PRÉ) das plantas daninhas e da cultura, pós-emergência (PÓS) da cultura e das plantas daninhas (CONSTANTIN, 2011) e ainda em pré-plantio de mudas pré-brotadas (SILVA et al., 2018). Segundo Victoria Filho e Christoffoleti (2004), em geral, no cultivo da cana-de-açúcar, os herbicidas são aplicados em PRÉ e/ou PÓS, com aplicação em PPI de uso restrito. Contudo a aplicação em PPI apresenta algumas vantagens, para "cana-planta" por exemplo, como evitar plantas daninhas em estádios de desenvolvimento avançado no momento da aplicação em PÓS (KUVA; SALVADO, 2014).

Dentre os principais herbicidas utilizados em áreas de plantio de cana-de-açúcar, pode-se destacar o sulfentrazone (CARBONARI et al., 2016). Esta molécula pertence ao grupo químico das triazolinonas, é um herbicida sistêmico, de alta solubilidade, e sua sorção no solo é classificada como de moderada a fraca. Apresenta registro para o controle de diferentes espécies de plantas daninhas nas culturas de cana-de-açúcar, soja, citros e café (MAPA, 2019) e é seletivo a estas culturas quando utilizado em pré-emergência (RODRIGUES; ALMEIDA, 2018). A absorção ocorre principalmente de forma radicular, e seu mecanismo de ação acontece através da inibição da enzima protoporfirinogênio oxidase (PROTOX) (OLIVEIRA JÚNIOR, 2011).

Estudos de Jones e Griffin (2008), Faria et al. (2014), Cerdeira et al. (2015), Dias et al. (2017) e Silva et al. (2018) avaliaram os efeitos da aplicação de sulfentrazone em cana-de-açúcar e verificaram que a seletividade pode variar com o modo de aplicação, dose do herbicida, textura do solo ou ainda com a variedade. As variedades CTC série 9000 e CTC 14 apresentam porte de crescimento ereto, período de colheita precoce para CTC 9001 e CTC 9003, médio para a CTC 9004 e tardio para CTC 14 (CTC, 2018). Assim, informações sobre a seletividade de herbicidas sobre as diferentes variedades de cana-de-açúcar são necessárias para um manejo de plantas daninhas eficaz, proporcionando a manutenção do potencial produtivo da variedade.

Assim, o objetivo do presente trabalho foi avaliar a seletividade do herbicida sulfentrazone, aplicado em pré-plantio incorporado e em préemergência de quatro variedades de cana-deaçúcar: CTC 9001, CTC 9002, СTC 9003 e CTC 14.

\section{Material e Métodos}

Os experimentos foram conduzidos em casa-de-vegetação, no município de Piracicaba $\mathrm{SP}$, em período compreendido entre setembro e novembro de 2015. Foram utilizadas quatro variedades de cana-de-açúcar centro 9001, CTC 9002, CTC 9003 e CTC 14), com duas modalidades de aplicação (PRÉ e PPI), totalizando assim oito experimentos.

O delineamento experimental adotado em todos os experimentos foi inteiramente casualizado, com quatro repetições. Cada unidade experimental foi constituída por um vaso de $7 \mathrm{~L}$, preenchido com solo e adubado com $25 \mathrm{~g}$ de fertilizante formulado N-P-K (8-28-16). A análise física e química do solo utilizado nos experimentos está apresentada na Tabela 1.

Tabela 1. Análise física e química do solo utilizado. Piracicaba - SP, 2015.

\begin{tabular}{|c|c|c|c|c|c|c|c|c|c|c|}
\hline $\mathrm{pH}\left(\mathrm{CaCl}_{2}\right)$ & MO & $\mathrm{Al}$ & $\mathrm{H}+\mathrm{Al}$ & $P$ (resina) & K & $\mathrm{Ca}$ & $\mathrm{Mg}$ & SB & CTC & V \\
\hline 5,12 & 5,05 & 0,6 & 20,0 & 4,0 & 1,2 & 15,0 & 6 & 22,2 & 42,2 & 53 \\
\hline \multicolumn{3}{|c|}{ Argila } & \multicolumn{3}{|c|}{ Silte } & \multicolumn{5}{|c|}{ Areia } \\
\hline \multicolumn{3}{|c|}{40} & \multicolumn{3}{|c|}{6} & \multicolumn{5}{|c|}{54} \\
\hline
\end{tabular}

Unidades: Al, $\mathrm{H}+\mathrm{Al}, \mathrm{K}, \mathrm{Ca}, \mathrm{Mg}$, SB e CTC $\left(\mathrm{mmol}_{\mathrm{c}} \mathrm{dm}^{-3}\right) ; \mathrm{P}$ (resina) $\left(\mathrm{mg} \mathrm{dm}{ }^{-3}\right) ; \mathrm{MO}\left(\mathrm{g} \mathrm{dm}^{-3}\right) ; \mathrm{V}(\%)$.

Os tratamentos constituíram-se da aplicação das doses $0,100,200,400,800$, e 1600 g i.a. ha ${ }^{-1}$ do herbicida sulfentrazone (Boral ${ }^{\circ} 500$ SC, FMC Agricultural Chemical Group, EUA). Ressalta-se que a dose máxima recomendada para cana-de-açúcar é de $800 \mathrm{~g}$ i.a. ha $^{-1}$ (RODRIGUES; ALMEIDA, 2018). Todas as aplicações foram realizadas em câmara de pulverização equipada com ponta tipo leque e jato plano, modelo XR 110.02, calibrada para 
proporcionar um volume de calda de $200 \mathrm{~L} \mathrm{ha}^{-1}$. Todos os vasos receberam uma lâmina de água proporcional a uma precipitação de $10 \mathrm{~mm}, 24$ horas após a aplicação. Durante o experimento a irrigação foi realizada por microaspersão, calculada para manutenção da umidade do solo em $70 \%$ da capacidade de campo, de forma homogênea.

Para a modalidade PRÉ, foi realizado o plantio dos toletes, a uma profundidade aproximada de 7-10 cm, com aplicação dos tratamentos em sequência. Em PPI, foi retirada uma camada em torno de 7-10 cm do solo de cada vaso, e em seguida foram colocados os toletes. $O$ solo retirado dos vasos foi acondicionado em caixas plásticas, e aplicados na câmara de pulverização. Em sequência, com o auxílio de sacos de plásticos, foi feita a homogeneização do solo, e logo após o mesmo foi recolocado sobre os toletes.

Foi realizada avaliação de fitotoxicidade aos 30, 37, 44, 51 e 58 dias após a aplicação (DAA), em que foram atribuídas notas percentuais a cada unidade experimental, onde 0 representa ausência de injúria e $100 \%$ a morte da planta (VELINI et al., 1995). Aos 58 DAA, foi coletada a parte aérea das plantas de cada um dos vasos. O material coletado foi seco em estufa com circulação de ar forçada, a 65으, por 72 horas, e posteriormente a massa seca das plantas de cana-de-açúcar foi mensurada em balança analítica com precisão de duas casas decimais.

Os dados obtidos para cada experimento foram submetidos à análise de variância ( $p \leq$ 0,05) (PIMENTEL-GOMES; GARCIA, 2002). As médias dos tratamentos foram comparadas pelo teste de Tukey (1949) a 5\% de probabilidade para as avaliações de fitotoxicidade. Quanto à variável massa seca, as médias foram submetidas à análise de regressão não-linear do tipo loglogístico, segundo o modelo proposto por Seefeldt et al. (1995).

$$
y=a+\frac{b}{1+\left(\frac{x}{c}\right)^{k}}
$$

Onde: $a$ é o limite inferior da curva;

$b$ é a diferença entre o limite superior e inferior da curva;

c é a dose em que reduz em $50 \%$ o acúmulo de massa seca;

$k$ é o coeficiente que indica a declividade da curva.

\section{Resultados \\ Fitotoxicidade \\ Sulfentrazone PRÉ}

Foram observados sintomas de fitotoxicidade nas quatro variedades de cana-deaçúcar, para aplicação de doses de sulfentrazone em PRÉ, sobretudo para as doses de 800 e $1600 \mathrm{~g}$ i.a. ha ${ }^{-1}$ (Tabela 2). Para a variedade CTC 9001, até a dose de $200 \mathrm{~g}$ i.a. ha ${ }^{-1}$, não foram verificados quaisquer sintomas, em todas as avaliações. No entanto, a partir da dose de $400 \mathrm{~g}$ i.a. $\mathrm{ha}^{-1}$, foram verificados sintomas de fitotoxicidade. Ao longo das avaliações, verificouse aumento nos sintomas de fitotoxicidade, com $16,3 \%, 43,8 \%$ e $61,3 \%$ para as doses de 400,800 e 1600 g i.a. ha ${ }^{-1}$, respectivamente aos 58 DAA.

$\mathrm{Na}$ variedade CTC 9002, foram observados sintomas em todas as avaliações a partir da dose de $400 \mathrm{~g}$ i.a. ha ${ }^{-1}$. O aumento da fitotoxicidade foi proporcional ao aumento das doses (400, 800 e 1600 g i.a. ha ${ }^{-1}$ ), em todas as avaliações. A variedade CTC 9003 foi tolerante à aplicação de sulfentrazone até a dose de $200 \mathrm{~g}$ i.a. ha ${ }^{-1}$. Contudo, maiores sintomas foram observados para a dose de $1600 \mathrm{~g}$ i.a. ha ${ }^{-1}$ a partir dos 37 DAA, com fitotoxicidade superior a $80 \%$. Enquanto que para a variedade CTC 14 foram notados sintomas de fitotoxicidade apenas a partir da dose de 800 g i.a. ha ${ }^{-1}$, e com maior injúria para a dose de 1600 g i.a. ha ${ }^{-1}$, com 72,5\% aos 58 DAA. 
Tabela 2. Fitotoxicidade (\%) das variedades de cana-de-açúcar CTC 9001, CTC 9002, CTC 9003 e CTC 14 para aplicação de doses de sulfentrazone em pré-emergência (PRÉ). Piracicaba - SP, 2015.

\begin{tabular}{|c|c|c|c|c|c|c|c|c|c|c|c|c|c|c|c|c|c|c|c|c|}
\hline \multirow{3}{*}{ Doses } & \multicolumn{20}{|c|}{ Dias após a aplicação } \\
\hline & \multicolumn{2}{|c|}{30} & \multicolumn{2}{|c|}{37} & \multicolumn{2}{|c|}{44} & \multicolumn{2}{|l|}{51} & \multicolumn{2}{|c|}{58} & \multicolumn{2}{|c|}{30} & \multicolumn{2}{|l|}{37} & \multicolumn{2}{|c|}{44} & \multicolumn{2}{|c|}{51} & \multicolumn{2}{|l|}{58} \\
\hline & \multicolumn{10}{|c|}{ СТС 9001} & \multicolumn{10}{|c|}{ CTC 9002} \\
\hline 0 & 0,0 & $a^{1}$ & 0,0 & a & 0,0 & $\mathrm{a}$ & 0,0 & a & 0,0 & a & 0,0 & a & 0,0 & A & 0,0 & $a$ & 0,0 & a & 0,0 & a \\
\hline 100 & 0,0 & $a$ & 0,0 & a & 0,0 & a & 0,0 & a & 0,0 & a & 0,0 & a & 0,0 & A & 0,0 & a & 0,0 & a & 5,0 & a \\
\hline 200 & 0,0 & $a$ & 1,3 & a & 0,0 & a & 0,0 & a & 0,0 & a & 0,0 & a & 0,0 & A & 0,0 & a & 0,0 & a & 5,0 & a \\
\hline 400 & 5,0 & b & 5,0 & $a b$ & 7,5 & $b$ & 8,8 & $b$ & 16,3 & b & 65,0 & $b$ & 65,0 & B & 55,0 & b & 47,5 & $b$ & 47,5 & $b$ \\
\hline 800 & 11,3 & c & 21,3 & b & 33,8 & c & 36,3 & c & 43,8 & c & 81,3 & c & 68,7 & B & 77,5 & c & 78,8 & c & 83,8 & c \\
\hline 1600 & 46,3 & d & 50,0 & C & 62,5 & d & 62,5 & d & 61,3 & d & 80,0 & C & 80,0 & C & 73,8 & C & 77,5 & C & 85,0 & C \\
\hline \multirow{2}{*}{$\begin{array}{r}\mathrm{CV} \\
(\%) \\
\end{array}$} & \multicolumn{2}{|c|}{21,2} & \multicolumn{2}{|c|}{21,6} & \multicolumn{2}{|c|}{17,7} & \multicolumn{2}{|c|}{16,8} & \multicolumn{2}{|c|}{18,6} & \multicolumn{2}{|c|}{12,0} & \multicolumn{2}{|c|}{10,9} & \multicolumn{2}{|c|}{16,0} & \multicolumn{2}{|c|}{19,2} & \multicolumn{2}{|c|}{12,1} \\
\hline & \multicolumn{10}{|c|}{ CTC 9003} & \multicolumn{10}{|c|}{ СТС 14} \\
\hline 0 & 0,0 & $a$ & 0,0 & $a$ & 0,0 & $a$ & 0,0 & $a$ & 0,0 & $a$ & 0,0 & a & 0,0 & a & 0,0 & $a$ & 0,0 & $a$ & 0,0 & a \\
\hline 100 & 0,0 & a & 0,0 & a & 0,0 & a & 0,0 & a & 0,0 & a & 0,0 & a & 0,0 & a & 0,0 & a & 0,0 & a & 0,0 & a \\
\hline 200 & 0,0 & a & 0,0 & $a$ & 0,0 & a & 0,0 & a & 0,0 & a & 2,3 & a & 0,0 & a & 0,0 & a & 0,0 & a & 0,0 & a \\
\hline 400 & 0,0 & $a$ & 5,0 & a & 10,0 & $b$ & 12,5 & $b$ & 16,3 & b & 3,8 & a & 5,0 & $a$ & 5,0 & $a$ & 0,0 & $a$ & 0,0 & $\mathrm{a}$ \\
\hline 800 & 28,8 & a & 31,3 & b & 33,8 & c & 37,5 & c & 37,5 & c & 55,0 & b & 35,0 & b & 45,0 & b & 33,8 & b & 36,3 & $b$ \\
\hline 1600 & 67,5 & $\mathrm{~b}$ & 82,5 & c & 90,0 & d & 90,0 & d & 87,5 & $d$ & 63,8 & C & 68,8 & C & 67,5 & C & 62,5 & C & 72,5 & C \\
\hline $\begin{array}{c}\mathrm{CV} \\
(\%)\end{array}$ & 19,7 & & 18 & & 18 & & 17,5 & & 16,4 & & 15 & & 12 & & 11,7 & & 10 & & 12,5 & \\
\hline
\end{tabular}

${ }^{1}$ Médias seguidas pela mesma letra minúscula na coluna não diferem entre si pelo teste de Tukey a $5 \%$ de probabilidade.

\section{Sulfentrazone PPI}

Foram verificados sintomas de fitotoxicidade para a aplicação de doses de sulfentrazone em PPI nas quatro variedades de cana-de-açúcar, principalmente a partir de $400 \mathrm{~g}$ i.a. ha ${ }^{-1}$ (Tabela 3).

Para as variedades CTC 9001 e CTC 9003, sintomas de fitotoxicidade ocorreram a partir da dose $400 \mathrm{~g}$ i.a. ha ${ }^{-1}$, considerados relativamente baixos, no máximo 7,5 e 12,5\% para CTC 9001 e CTC 9003, respectivamente. Entretanto, para a aplicação da maior dose de sulfentrazone $(1600 \mathrm{~g}$ i.a. ha $^{-1}$ ) foram verificados sintomas $\geq 90 \%$ para ambas as variedades.
A partir da dose de $200 \mathrm{~g}$ i.a. ha ${ }^{-1}$ foram observados sintomas de fitotoxicidade para a variedade CTC 9002, aos 30 DAA. Nas demais avaliações, a partir da dose de $400 \mathrm{~g}$ i.a. ha ${ }^{-1}$, aos 58 DAA, os sintomas foram de 35,50 e $76,3 \%$ para aplicação das doses 400, 800 e $1600 \mathrm{~g}$ i.a. $\mathrm{ha}^{-1}$, respectivamente. $\mathrm{Na}$ variedade CTC 14, foram verificados sintomas de fitotoxicidade apenas para as doses 800 e 1600 g i.a. ha ${ }^{-1}$, atingindo, aos 58 DAA, 86,3 e $90 \%$, respectivamente. 
Tabela 3. Fitotoxicidade (\%) das variedades de cana-de-açúcar CTC 9001, CTC 9002, CTC 9003 e CTC 14 para aplicação de doses de sulfentrazone em pré-plantio incorporado (PPI). Piracicaba - SP, 2015.

\begin{tabular}{|c|c|c|c|c|c|c|c|c|c|c|c|c|c|c|c|c|c|c|c|c|}
\hline \multirow{3}{*}{ Doses } & \multicolumn{20}{|c|}{ Dias após a aplicação } \\
\hline & \multicolumn{2}{|c|}{30} & \multicolumn{2}{|l|}{37} & \multicolumn{2}{|c|}{44} & \multicolumn{2}{|l|}{51} & \multicolumn{2}{|c|}{58} & \multicolumn{2}{|l|}{30} & \multicolumn{2}{|l|}{37} & \multicolumn{2}{|c|}{44} & \multicolumn{2}{|l|}{51} & \multicolumn{2}{|l|}{58} \\
\hline & \multicolumn{10}{|c|}{ СТC 9001} & \multicolumn{10}{|c|}{ CTC 9002} \\
\hline 0 & 0,0 & $a^{1}$ & 0,0 & a & 0,0 & $a$ & 0,0 & a & 0,0 & a & 0,0 & a & 0,0 & a & 0,0 & $a$ & 0,0 & a & 0,0 & a \\
\hline 100 & 0,0 & $a$ & 0,0 & a & 0,0 & $a$ & 0,0 & a & 0,0 & a & 0,0 & a & 0,0 & a & 0,0 & a & 0,0 & $a$ & 0,0 & a \\
\hline 200 & 0,0 & a & 5,0 & a & 5,0 & a & 0,0 & a & 0,0 & a & 17,5 & b & 6,3 & a & 5,0 & a & 5,0 & a & 5,0 & a \\
\hline 400 & 7,5 & b & 6,3 & a & 7,5 & $a$ & 7,5 & $\mathrm{~b}$ & 7,5 & b & 27,5 & c & 37,5 & b & 37,5 & b & 45,0 & b & 35,0 & b \\
\hline 800 & 20,0 & c & 51,3 & b & 56,3 & b & 56,3 & c & 60,0 & c & 53,8 & d & 32,5 & b & 42,5 & b & 41,3 & b & 50,0 & c \\
\hline 1600 & 66,3 & d & 85,0 & c & 90,0 & c & 92,5 & d & 92,5 & d & 91,3 & e & 82,5 & c & 82,5 & c & 80,0 & c & 76,3 & d \\
\hline \multirow{2}{*}{$\begin{array}{r}\text { CV } \\
(\%)\end{array}$} & 8,2 & & 14,8 & & 13 & & 7,5 & & 6,3 & & 10,2 & & 16 & & 18 & & 22,1 & & 17,4 & \\
\hline & \multicolumn{10}{|c|}{ СТС 9003} & \multicolumn{10}{|c|}{ CTC 14} \\
\hline 0 & 0,0 & a & 0,0 & $a$ & 0,0 & $a$ & 0,0 & a & 0,0 & a & 0,0 & a & 0,0 & a & 0,0 & $a$ & 0,0 & $a$ & 0,0 & a \\
\hline 100 & 0,0 & a & 0,0 & a & 0,0 & a & 0,0 & a & 0,0 & a & 0,0 & a & 0,0 & a & 0,0 & a & 0,0 & $a$ & 0,0 & a \\
\hline 200 & 0,0 & a & 0,0 & a & 0,0 & a & 0,0 & a & 0,0 & a & 0,0 & a & 0,0 & a & 0,0 & a & 0,0 & a & 0,0 & a \\
\hline 400 & 10,0 & b & 11,3 & b & 12,5 & b & 8,8 & a & 11,3 & b & 0,0 & a & 0,0 & a & 0,0 & a & 0,0 & a & 0,0 & $a$ \\
\hline 800 & 16,3 & c & 25,0 & c & 37,5 & c & 36,3 & b & 32,5 & c & 32,5 & b & 66,3 & b & 75,0 & b & 80,0 & b & 86,3 & b \\
\hline 1600 & 40,0 & d & 72,5 & d & 72,5 & d & 78,8 & c & 90,0 & d & 65,0 & c & 86,3 & c & 82,5 & b & 91,3 & c & 90,0 & b \\
\hline $\begin{array}{l}\text { CV } \\
\text { (\%) }\end{array}$ & 9,2 & & 15,6 & & 10 & & 9,7 & & 12,7 & & 9,6 & & 8,1 & & $13, \varepsilon$ & & 10,8 & & 7,1 & \\
\hline
\end{tabular}

${ }^{1}$ Médias seguidas pela mesma letra minúscula na coluna não diferem entre si pelo teste de Tukey a $5 \%$ de probabilidade.

\section{Massa seca}

\section{Sulfentrazone PRÉ}

Nas análises da massa seca da parte aérea das plantas de cana-de-açúcar, foram observadas que todas as variedades foram afetadas negativamente pela aplicação dos herbicidas em pré-emergência (Figura 1). A variedade CTC 9002 foi a mais sensível a aplicação de sulfentrazone em PRÉ, com redução da massa seca em $50 \%$ a partir da dose de 326,93 g i.a. ha ${ }^{-1}$, de acordo com a equação. Por outro lado, a variedade CTC 14 foi a mais tolerante, tendo $50 \%$ da massa seca prejudicada a partir da dose de 839,43 g i.a. ha ${ }^{-1}$. 
Figura 1. Redução do acúmulo de matéria seca das variedades de cana-de-açúcar CTC 9001, CTC 9002, CTC 9003 e CTC 14 para aplicação de doses de sulfentrazone em pré-emergência. Piracicaba - SP, 2015.

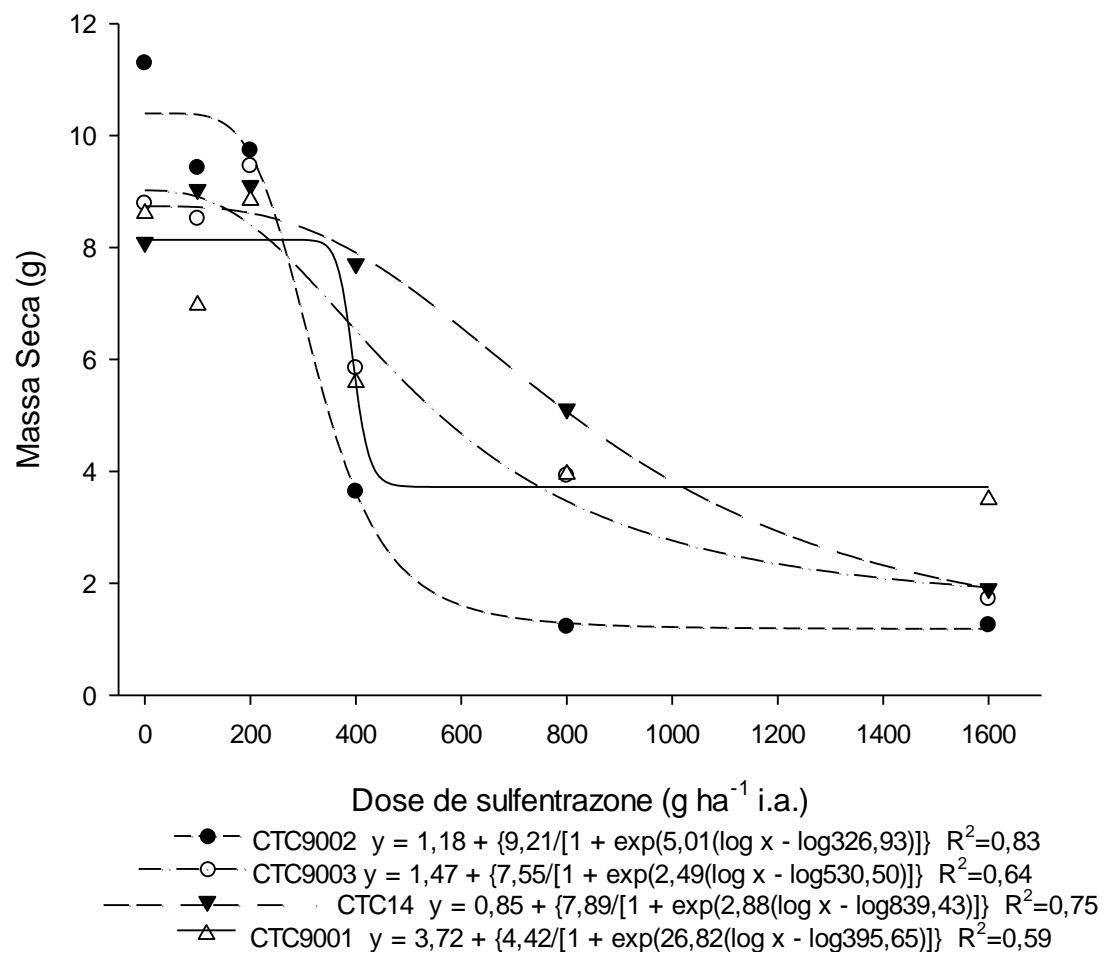

As doses de sulfentrazone aplicadas no estudo em PRÉ prejudicaram mais a CTC 14 com massa seca acumulada de $8,74 \mathrm{~g}$ e nas maiores doses aproximadamente $0,85 \mathrm{~g}$. A CTC 9002 foi capaz de acumular mais massa seca $(10,39 \mathrm{~g})$ e a CTC9001, mesmo nas maiores doses, teve a massa seca menos afetada ( $3,72 \mathrm{~g})$ se comparada às outras variedades. Comparando as quatro variedades, o acúmulo da massa seca foi CTC 9002 > CTC 9003 > CTC 14 > CTC 9001.

\section{Sulfentrazone PPI}

$\mathrm{Na}$ aplicação em PPI, o sulfentrazone afetou mais a CTC 14, e nas maiores doses (800 e $1600 \mathrm{~g}^{\text {i.a. }}$ ha $^{-1}$ ) acumularam menor massa seca (aproximadamente 0,42 g) (Figura 2). Todavia, a massa seca da variedade CTC 9002 foi a menos prejudicada nas maiores doses, com maior acúmulo de massa seca $(4,44 \mathrm{~g})$. 
Figura 2. Redução do acúmulo de matéria seca das variedades de cana-de-açúcar CTC 9001, CTC 9002, CTC 9003 e CTC 14 para aplicação de doses de sulfentrazone em pré-plantio incorporado. Piracicaba - SP, 2015.

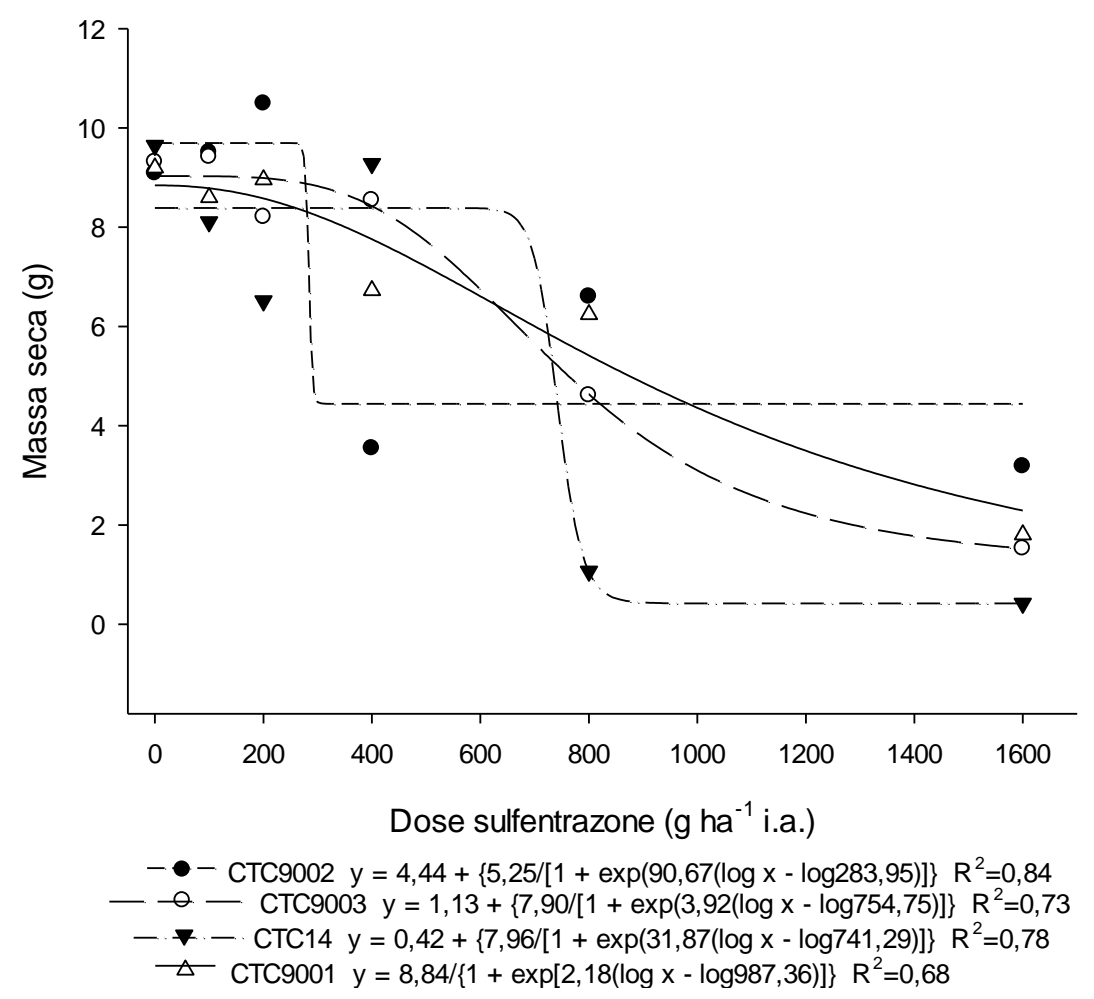

A CTC 9001 teve redução em 50\% da massa seca a partir da dose de 987,36 g i.a. ha ${ }^{-1}$, sendo a variedade mais tolerante a aplicação de sulfentrazone em PPI. De acordo com o coeficiente de declividade da equação $(2,18)$, a CTC 9001 é a variedade que sofre menor impacto na massa seca com a alteração da dose do sulfentrazone em PPI. No entanto, a CTC 9002 tem redução em $50 \%$ da massa seca a partir da dose de 283,85 g i.a. ha ${ }^{-1}$, se mostrando mais sensível.

\section{Discussão}

Dias et al. (2017) verificaram fitotoxicidade decorrente da aplicação de sulfentrazone (800 g i.a. ha ${ }^{-1}$ ) em pré-plantio de mudas pré-brotadas de cana-de-açúcar aos 28 DAA nas variedades CTC 14, CTC 7 e RB966928, com sintomas visuais de $30,26,5$ e $20 \%$, respectivamente. Apesar dos sintomas de fitotoxicidade, não foi verificada redução da massa seca da parte aérea das variedades, contudo, a CTC 14 foi mais tolerante ao sulfentrazone, com maior acúmulo de massa seca da parte aérea. Bertolino e Alves (2014), ao aplicarem sulfentrazone (700 g i.a. ha ${ }^{-1}$ ) em préemergência da variedade de cana-de-açúcar
SP81-3250, relataram que não houve injúria sobre a parte aérea, analisando separadamente a massa seca das folhas e do caule.

Em outro estudo, Correia (2016) observou sintomas de no máximo 3,3\% para aplicação de sulfentrazone (900 g i.a. ha ${ }^{-1}$ ) em "cana soca" após a colheita, nas variedades RB855453, RB835054 e RB867515, aos 30 e 60 DAA. Nesta situação, menores sintomas podem ser atribuídos pela aplicação ser em "cana soca", em que as plantas apresentam sistema radicular bem mais desenvolvido em relação ao presente estudo.

A aplicação de sulfentrazone (600 g i.a. $\mathrm{ha}^{-1}$ ) também provocou sintomas na variedade RB925345, com valor de $15 \%$ aos 54 DAA, para a aplicação em pós-emergência (Faria et al., 2014). Em avaliação de 120 DAA, Silva et al. (2018) também observaram sintomas de fitotoxicidade de até $30,83 \%$, em diferentes variedades de canade-açúcar, para aplicação de sulfentrazone $(800 \mathrm{~g}$ i.a. ha ${ }^{-1}$ ) em associação com ametryn (3000 g i.a. $h^{-1}$ ) ou metribuzin (1920 g i.a. ha ${ }^{-1}$ ) em pósplantio de mudas pré-brotadas.

No presente estudo, a partir da dose de $400 \mathrm{~g}$ i.a. ha ${ }^{-1}$ de sulfentrazone, foram observados sintomas de fitotoxicidade, com a intensidade 
alterando de acordo com a variedade. Estes resultados, e de outros estudos citados, reforçam que a seletividade e o potencial fitotóxico do sulfentrazone podem apresentar diferenças de acordo com a variedade de cana-de-açúcar, modo de aplicação, dose, sistema de plantio, textura do solo, características físico-químicas do solo como $\mathrm{pH}$, matéria orgânica, entre outros fatores.

Torres et al. (2012) observaram alterações em características fisiológicas (taxa transpiratória, eficiência de uso de água, taxa fotossintética e condutância estomática) em variedades de cana-de-açúcar submetidas à aplicação de sulfentrazone (600 g i.a. ha ${ }^{-1}$ ) e outros herbicidas (tembotrione, MSMA, diuron + hexazinone, trifloxysulfuron, tebuthiuron e clomazone) em pós-plantio, com alterações de acordo com a variedade. Este resultado reforça o fato que a seletividade ao sulfentrazone pode variar com a variedade de cana-de-açúcar, assim como observado por Ferreira et al. (2010) para aplicação de sulfentrazone e outros herbicidas (ametryn, tryfloxysulfuron, tebuthiuron e MSMA, por exemplo). A detoxificação do sulfentrazone pelo metabolismo da planta ocorre por degradação oxidativa através da enzima peroxidase (El Naggar et al., 1992), e é fator inerente a variedade e a capacidade desta em metabolizar o herbicida.

Zera et al. (2011) verificaram sintomas de fitotoxicidade ( 10\%) para aplicação de sulfentrazone (700 g i.a. ha ${ }^{-1}$ ) em pós-emergência das variedades IACSP94-2094, IACSP94-2101, IACSP93-3046, IACSP94-4004, IACSP86-2480, RB72454 e IACSP94-2101. Contudo, os percentuais de cada variedade foram muito próximos, e apesar dos sintomas de fitotoxicidade, não foram observadas reduções em produtividade e características tecnológicas (Brix, Pol, pureza do caldo, açúcares redutores, ATR).

O sulfentrazone é um herbicida seletivo à cana-de-açúcar, podendo ser aplicado até a dose de 800 g i.a. ha ${ }^{-1}$ (RODRIGUES; ALMEIDA, 2018). Possui baixa translocação e pode ser absorvido pelas raízes, caule e folhas de plantas jovens (ROMAN et al., 2007). A sorção do sulfentrazone no solo é dependente principalmente do teor de matéria orgânica $\left(K_{o c}=43\right)$ e pode ser também dependente do $\mathrm{pH}$ (FREITAS et al., 2009), além de possuir alto potencial de lixiviação $\left(\mathrm{S}_{\mathrm{w}}=780 \mathrm{mg} \mathrm{L}^{-}\right.$ $\left.{ }^{1}\right)$. Em estudo com sulfentrazone (750 g i.a. ha $\left.{ }^{-1}\right)$, Melo et al. (2010) relataram que em solo franco- arenoso com baixo e alto teor de matéria orgânica o sulfentrazone lixiviou até 27,5 e 17,5 $\mathrm{cm}$, respectivamente. Devido as características do herbicida, o sulfentrazone é indicado para aplicação em época seca (Procópio et al., 2008; Rodrigues e Almeida, 2018).

Dessa forma, compreende-se que o sulfentrazone aplicado em PRÉ apresenta menor fitotoxicidade e menor efeito sobre a massa seca das plantas de cana-de-açúcar com dissipação no solo, formando um gradiente de concentração de acordo com a profundidade. Todavia, ao aplicar em PPI, o sulfentrazone é colocado em maior concentração para ser absorvido diretamente na região de crescimento do sistema radicular da planta, causando maior fitointoxicação à cultura.

\section{Conclusões}

Nos experimentos em PRÉ e em PPI, o sulfentrazone foi fitotóxico à cana-de-açúcar a partir da dose de $400 \mathrm{~g}$ i.a. ha ${ }^{-1}$, sendo a CTC 14 a mais tolerante a aplicação do sulfentrazone nas duas modalidades de aplicação.

A CTC 9002 foi a variedade com maior acúmulo de massa seca tanto em PRÉ quanto em PPI, assim como a CTC 14 tolerou maiores doses do sulfentrazone em PRÉ, e a CTC 9001 em PPI.

\section{Referências}

BERTOLINO, C. B.; ALVES, P. L. D. C. A. Seletividade de herbicidas para cana-de-açúcar no sistema Plene em pré e pós-emergência. Revista Brasileira de Herbicidas, v.13, n.3, p.197-206, 2014. https://doi.org/10.7824/rbh.v13i3.249

CARBONARI, C. A.; GOMES, G. L. G. C.; TRINDADE, M. L. B.; SILVA, J. R. M.; VELINI, E. D. Dynamics of sulfentrazone applied to sugarcane crop residues. Weed Science, v.64, n.1, p.201206, 2016. https://doi.org/10.1614/ws-d-1400171.1

CERDEIRA, A. L.; PARAIBA, L. C.; QUEIROZ, S. C. N.; MATALLO, M. B.; FRANCO, D. A. S.; FERRACINI, V. L. Estimation of herbicide bioconcentration in sugarcane (Saccharum officinarum L.). Ciência Rural, n.45, n.4, p.591597, 2015. http://dx.doi.org/10.1590/0103$\underline{8478 \mathrm{cr} 20120466}$

CONSTANTIN, J. Mecanismos de Ação dos Herbicidas. In: OLIVEIRA JÚNIOR, R. S.; CONSTANTIN, J.; INOUE, M. H. Biologia e 
manejo de plantas daninhas. Curitiba: Ominipax, 2011. p. 67-68.

CORREIA, N. M. Chemical control of morning glory species in sugarcane harvested in the dry and semi-wet seasons. Planta Daninha, v.34, n.2, 333-343, 2016. http://dx.doi.org/10.1590/s0100$\underline{83582016340200015}$

CTC. Revista variedades CTC: Regional Piracicaba. Piracicaba: CTC, 2018.

DIAS, J. L. C. S.; SILVA JUNIOR, A. C.; QUEIROZ, J. R. G.; MARTINS, D. Herbicides selectivity in prebudded seedlings of sugarcane. Arquivos do Instituto Biológico, v.84, e0112015, 2017. http://dx.doi.org/10.1590/1808-1657000112015

EL NAGGAR, S. F.; CREEKMORE, R. W.; SCHOCKEN, M. J.; ROSEN, R. T.; ROBINSON, R. A. Metabolism of clomazone herbicide in soybean. Food Chemistry, v.40, p.880-883, 1992. http://dx.doi.org/10.1021/if00017a036

FARIA, A. T.; SARAIVA, D. T.; PEREIRA, A. M.; ROCHA, P. R. R.; SILVA, A. A.; SILVA, D. V.; FERREIRA, E. A.; SILVA, G. S. Herbicide effects on the atividadade rhizospheric microbial and growth of sugar cane. Bioscience Journal, v.30, n.4, p.1024-1032, 2014.

FERREIRA, R. R.; OLIVEIRA, F. T. R. D.; DELITE, F. D. S.; AZEVEDO, R. A.; NICOLAI, M.; CARVALHO, S. J. P. D.; CHRISTOFFOLETI, P. J.; FIGUEIRA, A. V. D. O. Differential tolerance of sugarcane varieties to herbicide stress. Bragantia, v.69, n.2, p.395-404, 2010.

http://dx.doi.org/10.1590/S0006-

87052010000200019

FREITAS, M. A. M.; PASSOS, A. B. R. J.; TORRES, L. G.; MORAES, H. M. F.; FAUSTINO, L. A.; ROCHA, P. R. R.; SILVA, A. A. Sorção do sulfentrazone em diferentes tipos de solo determinada por bioensaios. Planta Daninha, v.32, n.2, p.385-392, $2014 . \quad$ http://dx.doi.org/10.1590/5010083582014000200016

JONES, C. A.; GRIFFIN, J. L. Residual red morningglory (Ipomoea coccinea) control with foliar- and soil-applied herbicides. Weed Technology, v.22, n.3, 402-407, 2008. https://doi.org/10.1614/wt-07-099.1
KUVA, M. A.; PITELLI, R. A.; SALGADO, T. P.; ALVES, P. L. C. A. Phytosociology of weed community in no-burn sugar cane agroecosystems. Planta Daninha, v.25, n.3, p.501-511, 2007. http://dx.doi.org/10.1590/s0100$\underline{83582007000300009}$

KUVA, M. A.; SALGADO, T. P. Manejo de plantas daninhas nas principais culturas perenes do Brasil. Seção I: Manejo de plantas daninhas da cultura da cana-de-açúcar. In: MONQUERO, P. A. Manejo de plantas daninhas nas culturas agrícloas. São Carlos: RIMA - SBCPD, 2014. p. 82113.

MELO, C. A. D.; MEDEIROS, W. N.; TUFFI SANTOS, L. D.; FERREIRA, F. A.; TIBURCIO, R. A. S.; FERREIRA, L. R. Lixiviação de sulfentrazone, isoxaflutole e oxyfluorfen no perfil de três solos. Planta Daninha, v.28, n.2, p.385-392, 2010. http://dx.doi.org/10.1590/S010083582010000200017

OLIVEIRA JÚNIOR, R.S. Mecanismos de Ação dos Herbicidas. In: OLIVEIRA JÚNIOR, R. S.; CONSTANTIN, J.; INOUE, M. H. Biologia e manejo de plantas daninhas. Curitiba: Ominipax, 2011. p. 141-142.

PIMENTEL-GOMES, F.; GARCIA, C. H. Estatística aplicada a experimentos agronômicos e florestais: exposição com exemplos e orientações para uso de aplicativos. Piracicaba: Fealq, 2002.

PROCÓPIO, S. O.; SILVA, A. A.; VARGAS, L. Manejo e controle de plantas daninhas em canade-açúcar. In: VARGAS, L.; ROMAN, E. S. Manual de manejo e controle de plantas daninhas. 2.ed. Passo Fundo: EMBRAPA Trigo, 2008. 780 p.

RODRIGUES, B. N.; ALMEIDA. F. S. Guia de herbicidas, 7 ed. Londrina: Edição dos autores, 2018.

ROMAN, E. S.; BECKIE, H.; VARGAS, L.; HALL, L.; RIZZARDI, M. A.; WOLF, T. M. Como funcionam os herbicidas: da biologia à aplicação. Passo Fundo: Berthier, 2007.

SEEFELDT, S. S.; JENSEN, J. E.; FUERST, E. P. Loglogistic analysis of herbicide dose-response 
relationships. Weed Technology, v.9, n.2, p.218-

227, 1995.

https://doi.org/10.1017/S0890037X00023253

SILVA, T. P.; AZANIA, C. A. M.; XAVIER, M. A.; PERECIN, D., VITORINO, R. Sugarcane seedlings influenced by the management with herbicides. Planta Daninha, v.36, e018150678, 2018. http://dx.doi.org/10.1590/s0100$\underline{83582018360100019}$

TOLEDO, R. E. B.; SILVA JUNIOR, A. C.; NEGRISOLI, R. M.; NEGRISOLI, E.; CORRÊA, M. R.; ROCHA, M. G.; VICTORIA FILHO, R. Herbicidas aplicados em pré-emergência para o controle de Ipomoea spp. na cultura de cana-de-açúcar em época seca. Revista Brasileira de Herbicidas, v.14, n.4, p.271-279, 2015. https://doi.org/10.7824/rbh.v14i4.418

TORRES, L. G.; FERREIRA, E. A.; ROCHA, P. R. R.; FARIA, A. T.; GONÇALVES, V. A.; GALON, L.; SILVA, A. F.; SILVA, A. A. Changes in the physiological characteristics of sugarcane cultivars submitted to herbicide application. Planta Daninha, v.30, n.3, p.581-587, 2012. http://dx.doi.org/10.1590/S0100$\underline{83582012000300014}$

TUKEY, J. W. Comparing individual means in the analysis of variance. Biometrics, v.5, n.2, p.99114, 1949. https://doi.org/10.2307/3001913

VELINI, D.E.; OSIPE, R.; GAZZIERO, D. L. P. Procedimentos para instalação, avaliação e análise de experimentos com herbicidas. Londrina: SBCPD, 1995.

VICTORIA FILHO, R.; CHRISTOFFOLETI, P. J. Manejo de plantas daninhas e produtividade da cana. Visão Agrícola, n.1, 32-37, 2004.

ZERA, F. S.; AZANIA, C. A. M.; SCHIAVETTO, A. R.; LORENZATO, C. M.; AZANIA, A. A. P. M. Tolerance of different sugarcane (Saccharum spp.) cultivars to herbicides. Planta Daninha, v.29, n.3, p.591-599, 2011. http://dx.doi.org/10.1590/\$0100$\underline{83582011000300013}$ 